\title{
Transverse Relaxation of Scalar Coupled Protons in Magnetic Resonance of Non-Deuterated Proteins
}

\author{
Takuya F. Segawa $\cdot$ Bikash Baishya \\ Geoffrey Bodenhausen
}

Received: 13 October 2011/Published online: 20 November 2011

(C) Springer-Verlag 2011

\begin{abstract}
The transverse relaxation rates $R_{2}=1 / T_{2}$ of protons can be determined by spin-echo sequences with multiple refocusing pulses using moderate radio-frequency field strengths and properly chosen inter-pulse delays so as to suppress echo modulations due to homonuclear scalar couplings. Combination with $2 \mathrm{D}$ heteronuclear correlation spectroscopy (HSQC) allows one to measure $R_{2}$ of arbitrary protons attached to nitrogen-15 or carbon-13 nuclei. Decays of six amide protons in the protein Ubiquitin that is nitrogen-15 enriched (but not deuterated) were measured at different temperatures.
\end{abstract}

\section{Introduction}

It has been known since the 1950s that refocusing pulses fail to eliminate the effects of homonuclear scalar couplings and that echo trains are therefore

T. F. Segawa $\cdot$ B. Baishya $\cdot$ G. Bodenhausen

Laboratoire de Résonance Magnétique Biomoléculaire, Institut des Sciences et Ingénierie Chimiques, Ecole Polytechnique Fédérale de Lausanne, BCH, 1015 Lausanne, Switzerland

G. Bodenhausen $(\square)$

Département de Chimie, Ecole Normale Supérieure,

24 Rue Lhomond, 75231 Paris Cedex 05, France

e-mail: geoffrey.bodenhausen@ens.fr

G. Bodenhausen

UMPC, Paris, France

G. Bodenhausen

CNRS, UMR 7203, Paris, France

Present Address:

B. Baishya

Chemical Physics Department, Weizmann Institute, 76100 Rehovot, Israel 
modulated, thus making it difficult to determine transverse relaxation rates in coupled spin systems $[1,2]$. In 2006, we discovered a new method to quench echo modulations stemming from homonuclear scalar couplings [3]. When applying a Carr-Purcell-Meiboom-Gill (CPMG) $[1,2]$ sequence $(\pi / 2)_{y}-\left[\tau-\pi_{x}-\tau\right]_{2 n}$ to nitrogen-15 nuclei in RNA duplexes with scalar $J\left({ }^{15} \mathrm{~N},{ }^{15} \mathrm{~N}\right)$ couplings, the echo decays were found to be free of oscillations so that transverse relaxation rates could be readily determined. The crucial point of these experiments, i.e., the use of imperfect $180^{\circ}$ pulses with moderate radio-frequency (rf) amplitudes, was revealed by simulations: while the on-resonance spin $I$ undergoes a perfect rotation about $180^{\circ}$, the $J$-coupled off-resonance spin $S$ is rotated about a tilted field by an angle that exceeds $180^{\circ}$. In terms of product operators of a two-spin system, where spin $I$ is irradiated on resonance, the $J$ coupling normally tends to convert in-phase $I_{x}$ into anti-phase $2 I_{y} S_{z}$. Pulse imperfections bring an additional third product operator into the game, the multiple-quantum term $2 I_{y} S_{y}$. This leads to a stabilization of $I_{x}$, since $2 I_{y} S_{z}$ and $2 I_{y} S_{y}$ mutually inhibit each other's appearance. This interplay was called 'stabilization by interconversion within a triad of coherences under multiple refocusing', or SITCOM in short. A related effect had been observed 2 years earlier [4] while measuring transverse relaxation rates of multiple-quantum coherences $2 \mathrm{~N}_{x} \mathrm{H}_{x}$. The SITCOM effect can be considered as a form of decoupling. However, this effect fails when so-called 'recoupling conditions' are fulfilled. The $J$ modulation is reintroduced when the offset of the $J$-coupled off-resonance spin $S$ lies in the vicinity of a multiple of the pulse repetition rate:

$$
\Omega_{s} /(2 \pi) \approx k\left(2 \tau_{\mathrm{rec}}\right)^{-1}
$$

where $\Omega_{S} /(2 \pi)$ is the offset of spin $S, k$ an integer, and $2 \tau_{\text {rec }}$ the interval between two $180^{\circ}$ pulses when recoupling occurs. Eq. 1 has the same form as the sidebands in 'delays alternating with nutation for tailored excitation' (DANTE) [5-8]. Gopalakrishnan et al. [9] described these phenomena in terms of an average-Hamiltonian approach for a two-spin system. The average Hamiltonian has the isotropic form of the scalar coupling I.S when decoupling occurs, but is truncated to its weak form $2 I_{z} S_{z}$, when recoupling appears. For a two-spin system, the recoupling conditions depend on the rf amplitude $\omega_{1}$ of the $180^{\circ}$ pulses [10]:

$$
2 \tau_{\mathrm{rec}}=2\left(k \pi / \Omega_{\mathrm{s}}-\pi / 4 \omega_{1}\right)
$$

The SITCOM effect can be used to measure apparent transverse relaxation rates $R_{2}^{\text {app }}$ of homonuclear coupled carbon-13 nuclei in glycine [10] and in alanine enriched with both carbon-13 and nitrogen-15, where a large number of recoupling conditions make it difficult to obtain unmodulated echo decays [11]. Shifting the rf carrier frequency offers a way to fully quench the modulations and to obtain apparent transverse relaxation rates $R_{2}^{\text {app }}$. In 2009 we extended the measurements of apparent transverse relaxation rates to protons in the oligopeptide Cyclosporin A (CsA) [12] where the protons form an extensive coupled spin system. Obviously, this is relevant for any organic molecule. From Eq. 1 or 2 we can derive the interval between two neighboring recoupling conditions: 


$$
\Delta \tau_{\mathrm{rec}}=\pi / \Omega_{s}
$$

Thus, the closer the chemical shifts $\Omega_{S}$ of the coupled spins $S$, the greater the separation between neighboring recoupling conditions. This makes the experiments much easier for protons than for nitrogen-15 or carbon-13.

Overlapping peaks cause problems, since proper integration of individual resonances becomes difficult. We have shown that the coherence can be transferred from protons to well-resolved neighboring carbon-13 spins, using a sequence for 'insensitive nuclei enhanced by polarization transfer' (INEPT) [13] immediately after a CPMG sequence with moderate rf amplitudes applied to the protons [14]. This made overlapping methyl resonances in CsA accessible. The proton decay rates $R_{2}^{\text {app }}$ derived from INEPT and direct experiments are not exactly the same, since INEPT focuses on protons that are coupled to carbon-13, which have decays that are accelerated by dipolar ${ }^{1} \mathrm{H}-{ }^{13} \mathrm{C}$ interactions.

We have also shown that the use of $360^{\circ}$ or $720^{\circ}$ pulses instead of $180^{\circ}$ refocusing pulses in CPMG schemes could fully eliminate recoupling conditions [15].

Recently Barrère et al. [16] described an alternative method to measure echo decays that are not masked by modulations in homonuclear-coupled systems. In contrast to our SITCOM approach, their method is based on refocusing of the homonuclear $J$ couplings, in analogy to the 'perfect echo' of Takegoshi et al. [17]. Both sequences were independently designed to generate 'planar mixing' (with an effective zero- or double-quantum Hamiltonian) to reduce multiplet complexity in COSY experiments [18] and to observe spin waves by NMR [19]. Since they use only hard pulses, various schemes for refocusing homonuclear $J$ couplings are inherently broadband, but in principle limited to two-spin AX- or AB-systems. By contrast, SITCOM is selective, but has no limitations concerning the extent of the coupling networks. Since refocusing of homonuclear $J$ couplings $[16,17]$ does not prevent anti-phase magnetization from building up, the apparent transverse relaxation rates obtained are generally mixtures of relaxation rates of in-phase $I_{x}$ and anti-phase $I_{\mathrm{y}} S_{\mathrm{z}}$ terms. By contrast, the SITCOM approach can measure the decay rate of a pure in-phase $I_{x}$ coherence.

The study of internal dynamics in proteins $[20,21]$ usually relies on accurate measurements [22] of Overhauser effects ${ }^{15} \mathrm{~N}\left\{{ }^{1} \mathrm{H}\right\}$, combined [23, 24] with longitudinal relaxation rates $R_{1}\left({ }^{15} \mathrm{~N}\right)=1 / T_{1}\left({ }^{15} \mathrm{~N}\right)$ and transverse relaxation rates $R_{2}\left({ }^{15} \mathrm{~N}\right)=1 / T_{2}\left({ }^{15} \mathrm{~N}\right)$. Recently, longitudinal relaxation rates of two-spin order terms $2 \mathrm{~N}_{z} \mathrm{H}_{z}$ [25] and transverse relaxation rates of multiple-quantum coherences $2 \mathrm{~N}_{x} \mathrm{H}_{x}$ have also been investigated [4, 26-30]. So far, however, measurements of longitudinal and transverse relaxation rates of protons in proteins have by and large remained elusive. Early attempts [31] used selective pulses to avoid echo modulations. Spin-locking experiments that focus on the $R_{1 \rho}$ rates of amide $\mathrm{H}^{\mathrm{N}}$ protons in deuterated proteins were designed [32] and applied inter alia to study effects of molecular oxygen [33]. Studies of $R_{1 \rho}$ can also be carried out in proteins [34]. Such experiments allow one to study the 'dispersion' of the relaxation rates as a function of the rf amplitude. If one uses CPMG sequences, perturbations stemming from homonuclear $J$ couplings can be neglected under certain 
circumstances [35] or can be avoided by studying back-exchanged amide $\mathrm{H}^{\mathrm{N}}$ protons in deuterated proteins [36].

Unlike $R_{1 \rho}$ spin-locking experiments that focus on amide $\mathrm{H}^{\mathrm{N}}$ protons, our experiments do not suffer from any TOCSY effects [37], since there can be no coherence transfer between the on-resonance $\mathrm{H}^{\mathrm{N}}$ protons and the coupling partners that experience tilted effective fields. Likewise, our approach avoids the pitfalls of unwanted ROESY effects [38, 39]. However, our experiments do not allow one to study the 'dispersion' of relaxation rates as a function of pulse repetition frequency or effective rf amplitude.

The idea of combining our CPMG sequences with INEPT is sufficient to analyze all transverse proton relaxation rates of an oligopeptide like CsA. It can be adapted for $\mathrm{H}^{\mathrm{N}}$ protons by transferring their magnetization to ${ }^{15} \mathrm{~N}$ spins. However, in small proteins such as Ubiquitin, this strategy fails by lack of resolution and sensitivity of ${ }^{15} \mathrm{~N}$ spectra.

This work shows how this problem can be alleviated using heteronuclear singlequantum correlation spectroscopy (HSQC) [40-43] to monitor the amplitudes of proton echoes resulting from CPMG pulse trains with moderate rf amplitudes applied to the protons prior to the HSQC sequence. There are many variants of HSQC, any of which can be combined with our CPMG sequences without modification of the phase cycles, etc. The proton carrier frequency was set onresonance for the protons of interest during the CPMG scheme, and switched to the water resonance prior to the HSQC sequence. Refocusing pulses with moderate rf amplitudes were used during the CPMG trains, leading to tilted effective fields for the coupling partners, so as to quench echo modulations. The 2D signal amplitudes were determined using NMRPipe [44]. The resulting $\left[{ }^{1} \mathrm{H},{ }^{13} \mathrm{C}\right]$-CPMG-HSQC method allows one to confirm the apparent transverse relaxation rates $R_{2}^{\text {app }}\left({ }^{1} \mathrm{H}\right)$ of protons attached to ${ }^{13} \mathrm{C}$ in natural abundance in CsA that had previously been measured by CPMG-INEPT [14]. Since there were no significant differences in CsA, we switched our attention to ${ }^{15} \mathrm{~N}$-enriched (but not deuterated) Ubiquitin. If homonuclear scalar couplings act during the spin-echo train (i.e., if they are not quenched), anti-phase single-quantum coherences (SQC's) of the type $2 \mathrm{H}_{x}^{\mathrm{N}} \mathrm{H}_{z}^{\alpha}$ may appear at the end of the CPMG sequence. Such terms may be converted into $4 \mathrm{H}_{x}^{\mathrm{N}} \mathrm{H}_{y}^{\alpha} \mathrm{N}_{x}$ at the beginning of the $t_{1}$ interval of the HSQC sequence and interfere with the desired pathways. However, if the $\tau$ delays are chosen to quench the homonuclear $J$ modulations, only in-phase SQC terms such $\mathrm{H}_{y}^{\mathrm{N}}$ appear at the end of the CPMG echo train, so that a normal HSQC spectrum will be obtained by gradient selection.

A ${ }^{15} \mathrm{~N}$-labeled sample of Ubiquitin at $\mathrm{pH} 7.2$ in $9: 1 \mathrm{H}_{2} \mathrm{O}: \mathrm{D}_{2} \mathrm{O}$ was studied at $600 \mathrm{MHz}$. Using $\left[{ }^{1} \mathrm{H},{ }^{15} \mathrm{~N}\right]$-CPMG-HSQC, the rates $R_{2}^{\text {app }}\left({ }^{1} \mathrm{H}^{\mathrm{N}}\right)$ were measured for six residues (D32, G35, L50, L56, E64 and G76) (Fig. 1 solid curves). A typical $\left[{ }^{1} \mathrm{H},{ }^{15} \mathrm{~N}\right]-\mathrm{CPMG}-\mathrm{HSQC}$ spectrum is shown in Fig. 2. The modulated decays of ordinary spin echoes were recorded for comparison with a single refocusing $\pi$-pulse of duration $\tau_{\pi}$ applied at $T / 2=n\left(2 \tau+\tau_{\pi}\right)-\tau_{\pi} / 2$, followed by HSQC (Fig. 1 dashed curves). The proton $\pi$-pulses in both CPMG trains and experiments with a single refocusing pulse had an rf amplitude $v_{1}=6.6 \mathrm{kHz}$. In contrast to CsA, where $R_{2}^{\text {app }}<J\left(\mathrm{H}^{\mathrm{N}} \mathrm{H}^{\alpha}\right)$, one cannot resolve any modulations in Ubiquitin, since 

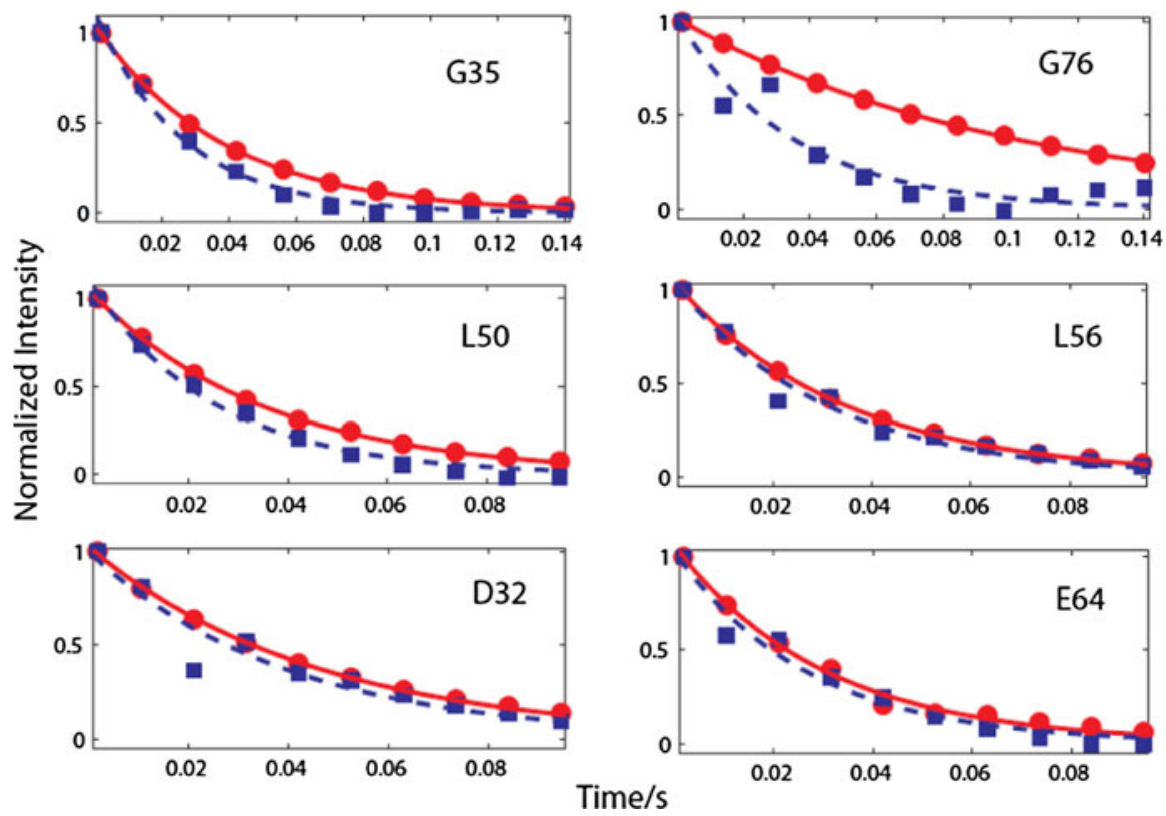

Fig. 1 Intensities of six different $\mathrm{H}^{\mathrm{N}}$ signals in Ubiquitin obtained by $\left[{ }^{1} \mathrm{H},{ }^{15} \mathrm{~N}\right]-\mathrm{CPMG}-\mathrm{HSQC}$ as solid a function of the duration $T=n\left(4 \tau+2 \tau_{\pi}\right)$ of echo trains with $\tau=400 \mu$ s at $300 \mathrm{~K}$ and $600 \mathrm{MHz}$. The increments were $n=1,8,16, \ldots, 80$ for G35 and G76, and $n=1,6,12, \ldots, 54$ for L50, L56, D32 and E64. The experimental points (circles) were fitted with monoexponential decays (solid curves). Squares show intensities of echoes that decay faster due to unresolved modulations that can be observed when using a single refocusing pulse with the same overall $T$ intervals, also followed by $\left[{ }^{1} \mathrm{H},{ }^{15} \mathrm{~N}\right]-\mathrm{HSQC}$. These values were also fitted with monoexponential decays (dashed curves) for comparison (Table 1). The proton $\pi$-pulses in the CPMG trains and in the experiments with single-refocusing pulses had the same rf amplitude $v_{1}=6.6 \mathrm{kHz}$

$R_{2}^{\text {app }}>J\left(\mathrm{H}^{\mathrm{N}} \mathrm{H}^{\alpha}\right)$, so that scalar modulations merely lead to faster decays (Table 1). The decay rate $R_{2}^{\text {app }}\left({ }^{1} \mathrm{H}^{\mathrm{N}}\right)$ of G76 is by far the slowest; this amino acid belongs to the flexible C-terminus of Ubiquitin so that only a few neighboring spins contribute to its dipolar relaxation.

For all residues except G76, the rates $R_{2}^{\text {app }}$ decrease with increasing temperature, as expected for exchange-free rates $R_{2}{ }^{\circ}$ since higher temperatures lead to shorter correlation times $\tau_{\mathrm{c}}$ [45]. The opposite trend of G76 can be attributed to the rate of exchange of the $\mathrm{H}^{\mathrm{N}}$ proton with water that increases with temperature.

The quenching of echo modulations in proteins is surprisingly straightforward. The largest offset $3.49 \mathrm{kHz}$ in E64 corresponds to an effective field tilted by $\arctan (\gamma)=28^{\circ}$ for $\mathrm{H}^{\alpha}(\mathrm{E} 64)$ when $\mathrm{H}^{\mathrm{N}}(\mathrm{E} 64)$ is on-resonance, since $\gamma=\Omega\left(\mathrm{H}^{\alpha}\right) /$ $\omega_{1}=0.53$ (see Table 1).

To verify that none of the CPMG-HSQC experiments suffered from accidental 'recoupling' effects at unfavorable $\tau$ intervals, a few 'hybrid' experiments [9] were carried out in 2D fashion (Fig. 3). For each residue, 101 spectra were recorded with the proton carrier frequency on-resonance, varying the delay $300<\tau<700 \mu$ s in steps of $4 \mu \mathrm{s}$, while keeping the number of $\pi$-pulses constant with $2 n=40$. An 


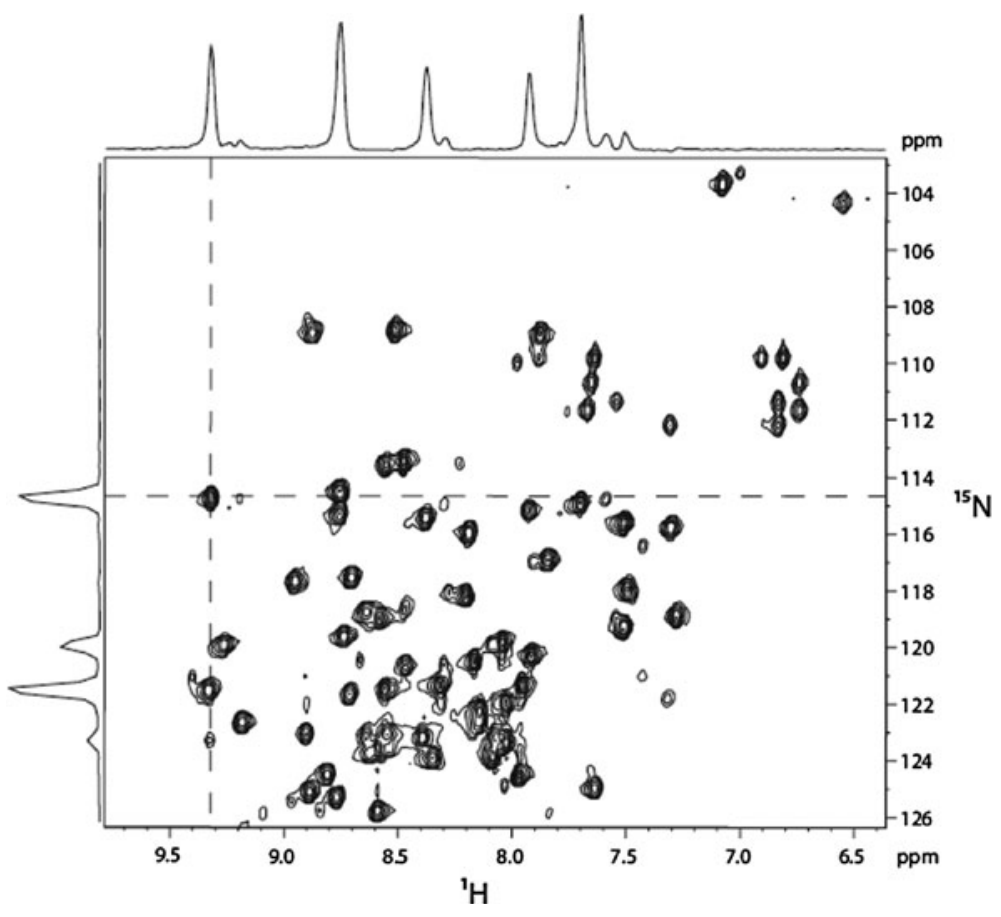

Fig. 2 Detail of a $\left[{ }^{1} \mathrm{H},{ }^{15} \mathrm{~N}\right]-\mathrm{CPMG}-\mathrm{HSQC}$ with cross sections (taken at the frequencies indicated by dashed lines) corresponding to E64 with $\delta\left({ }^{1} \mathrm{H}^{\mathrm{N}}\right)=9.31 \mathrm{ppm}$ and $\delta\left({ }^{15} \mathrm{~N}\right)=114.8 \mathrm{ppm}$, with $n=1$ (i.e., second echo) at $\mathrm{T}=315 \mathrm{~K}$. The number of transients was 4 , the recycle delay $3.5 \mathrm{~s}$, the number of points in the $t_{1}$ domain 72 , and the spectral width in the indirect $\omega_{1}$ dimension $26 \mathrm{ppm}$

experiment with a given offset covers several protons that have a similar chemical shift in the ${ }^{1} \mathrm{H}$ dimension of the HSQC spectrum. In this way, one hybrid HSQC can give hybrid plots for at least 10 amide proton resonances.

The vertical lines in Fig. 3 highlight the delay $\tau=400 \mu$ s where all CPMGHSQC spectra of Fig. 1 were recorded. Only for E64 does this coincide with a 'dip' due to echo modulations arising from 'recoupling', which explains the weak modulations of the circles in Fig. 1. This leads to a significant overestimation of $R_{2}^{\text {app }}$. This problem could be circumvented by repeating the CPMG sequence with another $\tau$ delay to avoid all 'recoupling dips', e.g., $\tau=480 \mu$ s. G76 stands apart not only by its slower decay rate, but also by a broader and deeper 'recoupling dip'. The position of these dips depends primarily on the chemical shifts of the coupling partners, and not on the scalar coupling constants, as can be seen from Eq. 2. Each 2D CPMG-HSQC spectrum took about $5 \mathrm{~min}$. The 2D experiments can be accelerated [46] by optimized aliasing [47], by SOFAST methods [48] or by singlescan $2 \mathrm{D}[49,50]$. Other protons in Ubiquitin could be studied by $\left[{ }^{1} \mathrm{H},{ }^{13} \mathrm{C}\right]-\mathrm{CPMG}-$ HSQC experiments correlating ${ }^{1} \mathrm{H}$ with ${ }^{13} \mathrm{C}$ nuclei through heteronuclear couplings $J\left({ }^{1} \mathrm{H},{ }^{13} \mathrm{C}\right)$, as we have done for $1 \mathrm{D}$ CPMG [14].

For the sake of demonstration, all CPMG experiments shown here were performed with the proton carrier on-resonance for the $\mathrm{H}^{\mathrm{N}}$ signals of interest. As 
Table 1 Apparent transverse proton relaxation rates $R_{2}^{\text {app }}\left({ }^{1} \mathrm{H}^{\mathrm{N}}\right)\left(\mathrm{s}^{-1}\right)$ in Ubiquitin, measured at three different temperatures at $600 \mathrm{MHz}$

\begin{tabular}{cccccrr}
\hline Temperature & D32 & G35 & L50 & L56 & E64 & G76 \\
\hline $285 \mathrm{~K}$ & $\mathbf{2 8 . 8}$ & $\mathbf{3 3 . 1}$ & $\mathbf{3 5 . 3}$ & $\mathbf{4 0 . 4}$ & $\mathbf{4 0 . 4}$ & $\mathbf{6 . 5}$ \\
& 43.3 & 58.4 & 90.4 & 63.0 & 94.1 & 30.2 \\
$300 \mathrm{~K}$ & $\mathbf{2 1 . 8}$ & $\mathbf{2 6 . 1}$ & $\mathbf{2 8 . 4}$ & $\mathbf{2 9 . 1}$ & $\mathbf{3 3 . 0}$ & $\mathbf{9 . 9}$ \\
& 25.1 & 37.9 & 40.0 & 32.6 & 37.0 & 28.9 \\
$315 \mathrm{~K}$ & $\mathbf{1 7 . 1}$ & $\mathbf{1 9 . 8}$ & $\mathbf{2 1 . 4}$ & $\mathbf{2 1 . 3}$ & $\mathbf{2 6 . 3}$ & $\mathbf{3 3 . 3}$ \\
& 19.4 & 34.7 & 35.1 & 23.9 & 32.1 & 40.5 \\
$\delta\left(\mathrm{H}^{\mathrm{N}}\right)(\mathrm{ppm})$ & 7.95 & 8.41 & 8.48 & 8.06 & 9.24 & 7.87 \\
$\delta\left(\mathrm{N}^{\mathrm{H}}\right)(\mathrm{ppm})$ & 119.8 & 108.9 & 125.7 & 118.0 & 114.6 & 119.1 \\
$\gamma=\Omega\left(\mathrm{H}^{\alpha}\right) / \omega_{1}$ & 0.33 & 0.40 & 0.40 & 0.37 & 0.53 & 0.37 \\
& & 0.42 & & & & 0.38 \\
\hline
\end{tabular}

The rates in boldface were obtained from monoexponential fits of unmodulated CPMG experiments (solid curves in Fig. 1), the rates in lightface stem from monoexponential fits of partly modulated echo envelopes obtained with a single refocusing pulse (dashed curves in Fig. 1). The shifts of the $\mathrm{N}^{\mathrm{H}}$ nitrogen-15 nuclei and the neighboring scalar-coupled $\mathrm{H}^{\mathrm{N}}$ protons are indicated for $T=300 \mathrm{~K}$, along with the ratio $\gamma$ that determines the tilt of the effective field experienced by the coupling partner $\mathrm{H}^{\alpha}$ if $\mathrm{H}^{\mathrm{N}}$ is on-resonance. All rates are associated with errors of $\pm 10 \%$
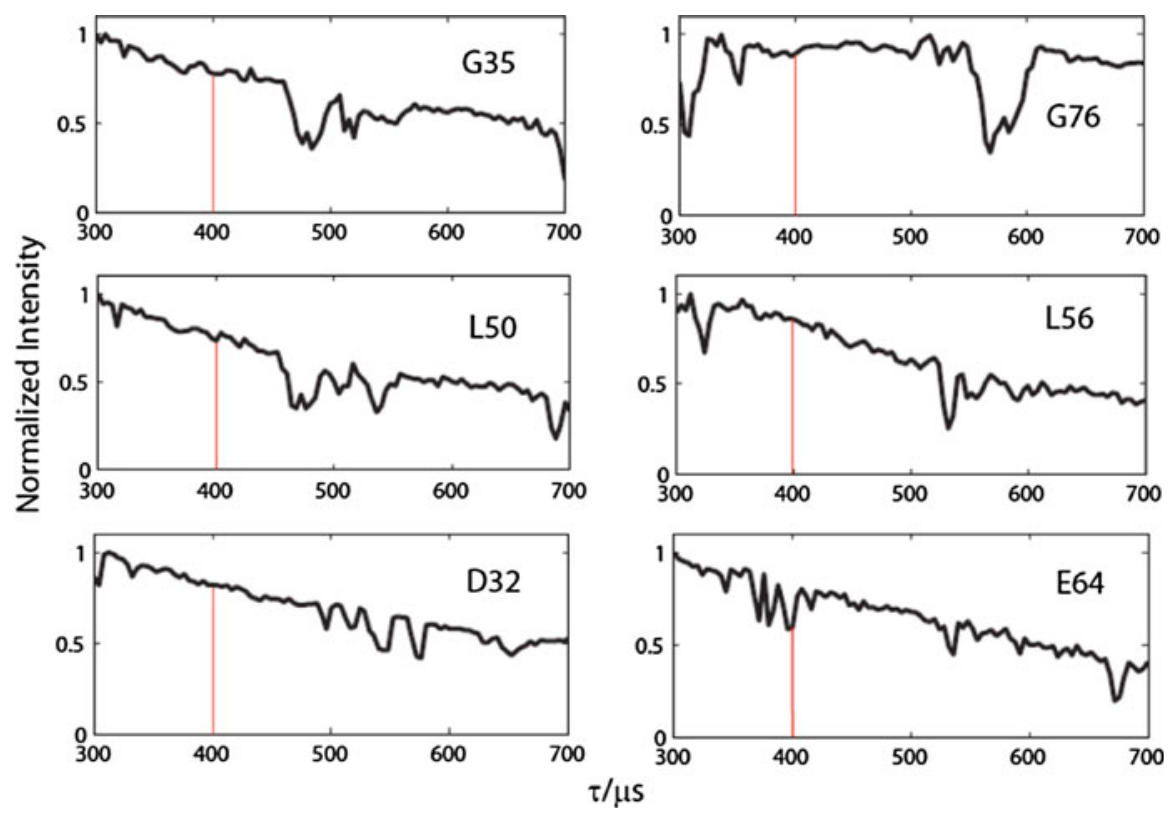

Fig. 3 Signals of $\mathrm{H}^{\mathrm{N}}$ protons in the backbone of Ubiquitin, obtained with the 'hybrid' version of [ ${ }^{1} \mathrm{H}$, ${ }^{15} \mathrm{~N}$ ]-CPMG-HSQC, where the number of refocusing pulses $2 n=40$ was kept constant, while the delay $\tau$ was incremented from 300 to $700 \mu \mathrm{s}$ in steps of $4 \mu \mathrm{s}$. The vertical lines correspond to the delay $\tau=400 \mu$ s used in Fig. 1 
recently demonstrated for systems with three carbon-13 nuclei [11], it is also possible to obtain unmodulated decays when the rf carrier is positioned offresonance. This allows one to measure the rates $R_{2}^{\text {app }}$ of different protons in a single experiment.

An alternative to CPMG with moderate rf field strengths would be the use of shaped pulses such as band-selective refocusing (REBURP) pulses [51] to achieve band-selective refocusing of all amide protons. However, such pulses would need to have a duration of about $3 \mathrm{~ms}$ to refocus all amide $\mathrm{H}^{\mathrm{N}}$ protons (ca $3 \mathrm{ppm}$ or $2 \mathrm{kHz}$ at $600 \mathrm{MHz}$ ) without affecting $\mathrm{H}^{\alpha}$ and other protons, in contrast to our scheme, where the magnetization of the coupling partners experiences tilted effective fields. It would be necessary to take into account various longitudinal and transverse relaxation processes during such protracted pulses, unless one chooses to use only a small number of band-selective refocusing pulses. In this case, these pulses would be separated by lengthy de- and refocusing intervals, and one would have to consider the 'contamination' of the transverse relaxation rates of in-phase terms $\mathrm{H}_{y}^{\mathrm{N}}$ by the decay rates of anti-phase coherences $2 \mathrm{H}_{x}^{\mathrm{N}} \mathrm{H}_{z}^{\alpha}$.

We have shown that a combination of multiple refocusing using moderate $\mathrm{rf}$ field-strengths and HSQC allows one to measure the apparent transverse relaxation rates $R_{2}^{\text {app }}$ of any proton that can be resolved in a $2 \mathrm{D}$ spectrum. Echo modulations due to omnipresent homonuclear couplings between protons can be quenched by cumulative pulse errors experienced by off-resonance coupling partners, due to the moderate strength of the refocusing pulses. This technique opens the way to measuring transverse relaxation rates of non-exchangeable protons such as $\mathrm{H}^{\alpha}, \mathrm{H}^{\beta}$, etc., in carbon-13-enriched proteins.

Acknowledgments We thank Martial Rey for technical support and are indebted to the Swiss National Science Foundation, the Commission for Technology and Innovation, the Ecole Polytechnique Fédérale de Lausanne and the CNRS for funding.

\section{References}

1. H.Y. Carr, E.M. Purcell, Phys. Rev. 94(3), 630-638 (1954)

2. S. Meiboom, D. Gill, Rev. Sci. Instrum. 29(8), 688-691 (1958)

3. J. Dittmer, G. Bodenhausen, Chem. Phys. Chem. 7(4), 831-836 (2006)

4. J. Dittmer, G. Bodenhausen, Chem. Phys. Chem. 5(11), 1750-1754 (2004)

5. G. Bodenhausen, R. Freeman, G.A. Morris, J. Magn. Reson. 23(1), 171-175 (1976)

6. G.A. Morris, R. Freeman, J. Magn. Reson. 29(3), 433-462 (1978)

7. R. Freeman, Chem. Rev. (Washington, DC, US) 91(7), 1397-1412 (1991)

8. R. Freeman, Prog. Nucl. Magn. Reson. Spectrosc. 32, 59-106 (1998)

9. K. Gopalakrishnan, N. Aeby, G. Bodenhausen, Chem. Phys. Chem. 8(12), 1791-1802 (2007)

10. N. Aeby, G. Bodenhausen, Chem. Phys. Lett. 463(4-6), 418-421 (2008)

11. T.F. Segawa, N. Aeby, G. Bodenhausen, Phys. Chem. Chem. Phys. 12(33), 9772-9776 (2010)

12. B. Baishya, T.F. Segawa, G. Bodenhausen, J. Am. Chem. Soc. 131(48), 17538-17539 (2009)

13. G.A. Morris, R. Freeman, J. Am. Chem. Soc. 101(3), 760-762 (1979)

14. T.F. Segawa, B. Baishya, G. Bodenhausen, Chem. Phys. Chem 11(15), 3343-3354 (2010)

15. B. Baishya, T.F. Segawa, G. Bodenhausen, J. Magn. Reson. 211(2), 240-242 (2011)

16. C. Barrère, P. Thureau, A. Thévand, S. Viel, Chem. Commun. (Cambridge, UK) 47(32), 9209-9211 (2011)

17. K. Takegoshi, K. Ogura, K. Hikichi, J. Magn. Reson. 84(3), 611-615 (1989) 
18. T. Schulte-Herbruggen, Z.L. Madi, O.W. Sorensen, R.R. Ernst, Mol. Phys. 72(4), 847-871 (1991)

19. Z.L. Madi, B. Brutscher, T. Schulte-Herbruggen, R. Bruschweiler, R.R. Ernst, Chem. Phys. Lett. 268(3-4), 300-305 (1997)

20. C. Wang, A.G. Palmer, Magn. Reson. Chem. 41(10), 866-876 (2003)

21. A. Mittermaier, L.E. Kay, Science 312(5771), 224-228 (2006)

22. F. Ferrage, D. Cowburn, R. Ghose, J. Am. Chem. Soc. 131(17), 6048-6049 (2009)

23. G. Lipari, A. Szabo, J. Am. Chem. Soc. 104(17), 4546-4559 (1982)

24. G. Lipari, A. Szabo, J. Am. Chem. Soc. 104(17), 4559-4570 (1982)

25. P. Pelupessy, F. Ferrage, G. Bodenhausen, J. Chem. Phys. 126(13), 134508 (2007)

26. J. Dittmer, G. Bodenhausen, J. Am. Chem. Soc. 126(5), 1314-1315 (2004)

27. V.Y. Orekhov, D.M. Korzhnev, L.E. Kay, J. Am. Chem. Soc. 126(6), 1886-1891 (2004)

28. M. Verde, S. Ulzega, F. Ferrage, G. Bodenhausen, J. Chem. Phys. 130(7), 074506 (2009)

29. S. Ulzega, M. Verde, F. Ferrage, G. Bodenhausen, J. Chem. Phys. 131(22), 224503 (2009)

30. S. Ulzega, N. Salvi, T.F. Segawa, F. Ferrage, G. Bodenhausen, Chem. Phys. Chem. 12(2), 333-341 (2011)

31. B. Boulat, G. Bodenhausen, J. Biomol. NMR 3(3), 335-348 (1993)

32. R. Ishima, P.T. Wingfield, S.J. Stahl, J.D. Kaufman, D.A. Torchia, J. Am. Chem. Soc. 120(40), 10534-10542 (1998)

33. T.S. Ulmer, I.D. Campbell, J. Boyd, J. Magn. Reson. 157(2), 181-189 (2002)

34. P. Lundstrom, M. Akke, J. Biomol. NMR 32(2), 163-173 (2005)

35. R. Ishima, D.A. Torchia, J. Biomol. NMR 25(3), 243-248 (2003)

36. D.M. Korzhnev, K. Kloiber, L.E. Kay, J. Am. Chem. Soc. 126(23), 7320-7329 (2004)

37. L. Braunschweiler, R.R. Ernst, J. Magn. Reson. 53(3), 521-528 (1983)

38. A. Bax, D.G. Davis, J. Magn. Reson. 63(1), 207-213 (1985)

39. C. Griesinger, G. Otting, K. Wuthrich, R.R. Ernst, J. Am. Chem. Soc. 110(23), 7870-7872 (1988)

40. G. Bodenhausen, D.J. Ruben, Chem. Phys. Lett. 69(1), 185-189 (1980)

41. A.G. Palmer, J. Cavanagh, P.E. Wright, M. Rance, J. Magn. Reson. 93(1), 151-170 (1991)

42. L.E. Kay, P. Keifer, T. Saarinen, J. Am. Chem. Soc. 114(26), 10663-10665 (1992)

43. J. Schleucher, M. Schwendinger, M. Sattler, P. Schmidt, O. Schedletzky, S.J. Glaser, O.W. Sorensen, C. Griesinger, J. Biomol. NMR 4(2), 301-306 (1994)

44. F. Delaglio, S. Grzesiek, G.W. Vuister, G. Zhu, J. Pfeifer, A. Bax, J. Biomol. NMR 6(3), 277-293 (1995)

45. C. P. Slichter, Principles of magnetic resonance, Third enlarged and updated edn. (Springer, Berlin, 1996)

46. P. Schanda, Prog. Nucl. Magn. Reson. Spectrosc. 55(3), 238-265 (2009)

47. D. Jeannerat, J. Magn. Reson. 186(1), 112-122 (2007)

48. P. Schanda, B. Brutscher, J. Am. Chem. Soc. 127(22), 8014-8015 (2005)

49. L. Frydman, T. Scherf, A. Lupulescu, Proc. Natl. Acad. Sci. USA 99(25), 15858-15862 (2002)

50. P. Pelupessy, J. Am. Chem. Soc. 125(40), 12345-12350 (2003)

51. H. Geen, R. Freeman, J. Magn. Reson. 93(1), 93-141 (1991) 